\title{
Sinusitis in children and adolescents with chronic or recurrent headache: a case-control study
}

\author{
Nesrin Şenbil · Y. K. Yavuz Gürer • \\ Çiğdem Üner · Yaşar Barut
}

Received: 23 October 2007 / Accepted: 22 November 2007/Published online: 25 January 2008

(C) Springer-Verlag 2008

\begin{abstract}
The aim of this study was to determine the frequency of misdiagnosis of sinus headache in migraine and other primary headache types in the children and adolescents with chronic or recurrent headaches. Children with chronic or recurrent headaches $(n=310)$ were prospectively evaluated. Data collection for each patient included history of previously diagnosed sinusitis due to headache, and additional sinusitis complaints (such as fever, cough, nasal discharge, postnasal discharge) at the time of sinusitis diagnosis, and improvement of the headache following treatment of sinusitis. If sinus radiographs existed they were recorded. The study included 214 patients with complete data. One hundred and sixteen (54.2\%) patients have been diagnosed as sinusitis previously and $25 \%$ of them had at least one additional complaint, while $75 \%$ of them had none. Sinusitis treatment had no effect on the headaches in $60.3 \%$ of the patients. Sinus graphy had been performed in $52.8 \%$, and $50.4 \%$ of them were normal. The prevalence of sinus headache concomitant with primary headache, and only
\end{abstract}

N. Şenbil · Y. K. Y. Gürer

Department of Child Neurology,

Dr. Sami Ulus Children's Hospital, Ankara, Turkey

N. Şenbil ( $\square)$

Fuar Sokak No: 7/9 Y. Ayranc1, Ankara, Turkey

e-mail: senbilon@yahoo.com

Ç. Üner

Department of Radiology,

Dr. Sami Ulus Children's Hospital, Ankara, Turkey

Y. Barut

Department of Otolaryngology,

Dr. Sami Ulus Children's Hospital, Ankara, Turkey sinus headache was detected in 7 and $1 \%$, respectively, in our study. Approximately $40 \%$ of the patients with migraine and $60 \%$ of the patients with tension-type headache had been misdiagnosed as "sinus headache". Children with chronic or recurrent headaches are frequently misdiagnosed as sinus headache and receive unnecessary sinusitis treatment and sinus graphy.

Keywords Headache $\cdot$ Migraine $\cdot$ Sinusitis

\section{Introduction}

The American Migraine Study revealed that nearly 4 million individuals are meeting International Headache Society's (IHS) migraine criteria and $14 \%$ of all migraine sufferers in the United States are diagnosed with sinus or tension-type headaches, but not with migraines [1]. The high frequency of sinus headache, which specialists consider to be relatively rare, among patients meeting IHS diagnostic criteria for migraine raises the possibility that migraine and perhaps other headache types are sometimes misdiagnosed as sinus headache [2].

The American Academy of Otolaryngology-Head and Neck Surgery (AAO-HNS) nominated a task force to produce working definitions for acute, subacute, and chronic rhinosinusitis in adults. They defined headache as a minor factor in the diagnosis of rhinosinusitis, and facial pain/pressure alone was not considered suggestive of a history of rhinosinusitis in the absence of another major nasal symptom or sign [3]. The American Academy of Pediatrics published clinical practice guidelines for the management of sinusitis in which headache is a variable complaint for the diagnosis of acute, subacute, and recurrent acute bacterial sinusitis in children [4]. The IHS 
system is only interested in acute sinus headache. The Headache Classification Committee commented "Chronic sinusitis is not validated as a cause of headache unless relapsing into an acute stage" in the IHS-1 criteria [5] and in the second revision of the International Classification of Headache Disorders Criteria (ICHD-II) [6].

Sinus-related pain or headaches have not been thoroughly studied until to date. The aim of this study was to determine the frequency of misdiagnosis of sinus headache in migraine and other primary headache types in the children with chronic or recurrent headaches.

\section{Methods}

The children referred to our pediatric neurology clinic, which is a tertiary step medical center in our country, with chronic or recurrent headaches $(n=310)$ were prospectively evaluated between February 2001 and February 2004. Children with a history of headache for at least 3 months and/or who had experienced at least 5 headache attacks (or 2 attacks in cases of migraine with aura) were included in this study. Upon physical and neurological examinations, they were asked to answer a questionnaire, with the help of a family member, on the characteristics of their headaches. The questionnaire included questions about the date of onset, recent frequency, the number of headache attacks, duration of headaches, pain features, headache triggering and ending factors, and accompanying symptoms, which also involved IHS-I criteria [5], and some other additional information. Upon completion of the medical history, and physical and neurological examinations, if necessary, additional investigations were conducted. Finally, the diagnoses were established in the light of the histories, examinations, headache questionnaires, headache diaries, and follow-up according to IHS-I criteria [5] and International Headache Society-Revised (IHS-R) criteria for migraine [7].

We also asked all subjects if they were previously diagnosed as sinusitis due to headache by a physician, and if so, we asked if the headache was different than their present headache and if they had additional complaints such as fever, cough, nasal discharge, and postnasal discharge at the time of sinusitis diagnosis. We asked whether or not the treatment of the sinusitis had an effect on the headache. If a patient had sinus radiographs at the time of sinusitis diagnosis, they were recorded.

At admission or follow-up, when a patient was thought to have had a sinus headache they were examined by an otolaryngology specialist and paranasal computed tomography (CT) scans were performed on the patients with acute sinusitis if their sinus-related headaches persisted in spite of appropriate treatment. Diagnoses of sinusitis were made in the light of the AAO-HNS task force recommendations and the American Academy of Pediatrics Clinical Practice Guideline: Management of Sinusitis [3,4].

\section{Results}

The study involved 214 patients (53.7\% girls, $46.3 \%$ boys) for whom we had complete data and who did not have secondary headaches except sinus headache. The mean age of the patients was $10.8 \pm 2.6$ years (range 4-16 years), the mean duration of intermittent headache symptoms was $22.8 \pm 19$ months (range 2-120), and the range of followup was 3 months to 3 years.

Prior to admission to our clinic, 116 patients $(54.2 \%)$ had a history of sinusitis diagnosis, while 98 patients (45.8\%) had none. Twenty-five percent (29/116) of the patients with a history of sinusitis diagnosis had at least one additional complaints, but $75 \%$ of them (87/116) did not and they all had the same headache characteristics at time of sinusitis diagnosis. On the other hand; 87 patients who have been diagnosed as sinusitis previously had the same headache characteristics both at the time of the sinusitis diagnosis and at the admission to our clinic.

Sinusitis treatment had no effect on headaches in $60.3 \%$ (70/116) of the patients, a short-term effect in $8.6 \%$ (10/ $116)$, and a long-term effect in $31.1 \%$ (36/116).

Sinus radiography had been performed previously in $52.8 \%(113 / 214)$ of the patients. The results were as follows: $50.4 \%$ (57/113) normal, $28.3 \%$ (32/113) sinus opacification, $21.3 \%$ (24/113) mucosal thickening.

At admission or during follow-up, 26 patients were evaluated for suspected sinus headache. Eleven patients were not diagnosed as sinus headache. Six patients had normal and five had abnormal but unrelated paranasal CT findings. In the light of the evaluations, $7 \%$ of the patients $(15 / 214)$ (acute bacterial sinusitis $=9$, mucosal contact point headache $=3$, acute exacerbations of chronic sinusitis $=3$ ) were diagnosed as sinus headaches. Ten patients had headache characteristics fulfilling IHS-R criteria for migraine, while three of them also had another type of headache. Three patients had clinical migraines. Only sinus headache has been diagnosed in two patients $(1 \%)$.

The diagnosis of headache types in the study group were as follows: IHS-R migraine $=123$ (without aura $=82$, with aura $=41$ ), tension type $=28$, clinical migraine $=$ 24 , migraine and tension-type $=19$, idiopathic stabbing headache $($ ISH $)=6$, cluster $=1$, unclassifiable $=11$, and only sinus headache.

The patients with migraine or other types of headaches who had been previously diagnosed as sinusitis in spite of having the same headache features at the time of the 


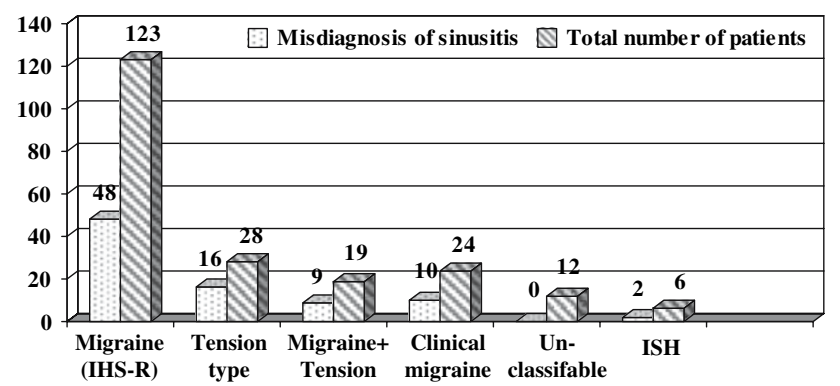

Fig. 1 The patients with primary headache who had been previously diagnosed as sinusitis in spite of having the same headache features at the time of the sinusitis diagnosis (misdiagnosis)

sinusitis diagnosis (misdiagnosis) are shown in Fig. 1. Sinus headache were determined in $8 \%(7 / 87)$ of these patients. Six patients had additional headache symptoms fulfilling IHS-R criteria for migraine, but one of them $(1.1 \%)$ had sinus headache only. In summary, 39\% (48/ 123) of the patients had headache characteristics fulfilling IHS-R criteria for migraine; $41.6 \%$ (10/24) had clinical migraine; $57.1 \%(16 / 28)$ had tension-type headache; $47.3 \%$ (9/19) had tension and migraine, and 33.3\% (2/6) had ISH.

\section{Discussion}

Approximately $40 \%$ of our patients fulfilled the IHS-R criteria for migraine and $60 \%$ of our patients with tensiontype headache had been previously misdiagnosed as "sinusitis". The results of our study are similar to the American Migraine Study II that was a follow-up study. In this study, 39.6\% of respondents meeting IHS diagnostic criteria for migraine reported that a physician had diagnosed them as sinus headache with or without additional headache types such as migraine or tension [1]. To our knowledge this is the first study which investigates misdiagnosed migraine as sinus headache in children and adolescents.

IHS-I criteria for migraine have poor sensitivity and a $26.9-66 \%$ rate of diagnostic capability in childhood [813]. In the study by Winner et al., a comparison of the diagnostic rates of pediatric migraine of the total sample revealed IHS (66\%) versus IHS-R (93\%) and the patients under 12 years of age had IHS (49\%) versus IHS-R (87\%) [7]. Therefore, we used the IHS-R criteria for migraine in the present study.

Although the $25 \%$ of patient who have been diagnosed as sinusitis previously had at least one sinusitis related complaint, this finding does not seem to be important because $60 \%$ of the patients said there had been no improvement after sinusitis treatment. Cady et al. reported that their patients were excluded if their recent headaches were associated with fever or purulent discharge, or if they had shown radiographic evidence of a sinus infection, and $98 \%$ of their patients had symptoms fulfilling the IHS criteria for migraine $(70 \%)$ or migraneous headache $(28 \%)$. Only $30 \%$ of their patients were satisfied with their headache therapy, whereas $63 \%$ were dissatisfied, and $7 \%$ felt neutral [14]. Most patients with a self-described sinus headache that had characteristics fulfilling IHS criteria for migraine or migraneous headache, also reported nasal symptoms and responded to migraine-specific therapy with sumatriptan [15]. A possible mechanism of sinus symptoms in migraine and vice versa can be explained by the hypothesized mechanism of neurogenic or immunogenic switching [4, 16]. Approximately $40 \%$ of our patients said their headache had been improved (short-term or longterm) after sinusitis treatment. This response may be irrelevant for episodic headache or there may be high placebo response or the child may respond to an analgesic given as part of the sinusitis treatment.

We investigated the histories of sinusitis that were diagnosed only by a doctor. These diagnoses were reported by patients and parents rather than obtained from physician's records. Therefore, this diagnosis in the past was neither true nor false. But, we included only the patients who had the same headache characteristics at the time of sinusitis diagnosis and at the admission, to evaluate misdiagnosis of sinusitis. The bias of this study was unsuccessfully treated patients who presented to our clinic, thus successfully treated sinus headache cases could have been overlooked.

Although the term sinusitis is traditionally preferred, mucosal linings of the nose and sinuses are simultaneously involved; therefore, the term rhinosinusitis is more appropriate [3]. Some authors have reported that AAO-HNS criteria are superior to IHS classifications and suggested that IHS classifications should be revised and improved with new diagnostic techniques [17]. "Sinus headache" are also modified and are renamed as "Headache attributed to rhinosinusitis" in the International Classification of Headache Disorders-Second Edition [6].

It may be quite difficult to differentiate the characteristics of sinus headache and other types of headaches, especially in the childhood. All our patients, except two with only the diagnosis of sinus headache suffered from migraines or another types of headaches. The characteristics of headaches were a tingling or leaking sensation, or they mimicked underlying migraine or other types of headaches. The number of our patients was limited to evaluate the characteristics of headaches in sinusitis or to compare them with the characteristics of other underlying headaches. Characteristics of sinus-related pain in chronic sinusitis patients were investigated in one study. The results of this study showed $38 \%$ of patients had persistent facial pain in spite of the lack of any evidence of persistent 
sinusitis, and there was no correlation between the severity of pain and the extent and location of mucosal disease. Furthermore, the site of pain did not correlate with the site of disease [18]. Isolated headache and facial pain and/or pressure are less reliable predictors than CT scans supporting the diagnosis of rhinosinusitis [19]. Further clinical studies investigating the characteristics of headaches in acute rhinosinusitis are needed.

Children with chronic or recurrent headaches are frequently misdiagnosed as sinus headache and patients receive unnecessary sinusitis treatment. Although patients presenting to pediatric neurology clinics with chronic or recurrent headache rarely have sinus headache alone, they may also have other headaches accompanied by sinusitis complaints. The characteristics of such patients should be critically evaluated. In particular, patients with treatment-resistant chronic headaches should be assessed for rhinosinusitis and mucosal contact point headache, even if they have the characteristics of migraine and other types of headaches. A paranasal CT scan and an examination by an otolaryngology specialist should be performed when needed.

In our study, approximately $40 \%$ of the patients with migraine and $60 \%$ of the patients with tension-type headache had been misdiagnosed as "sinus headache". Misdiagnosis of migraine is similar in our country to the United States. Most patients with headache are seen by primary care physicians and pediatricians who have little education on headache and migraine. Therefore, we suggest education regarding headache to improve diagnosis of primary headache and migraine among primary care physicians and pediatricians.

Conflicts of interest None.

\section{References}

1. Diamond ML (2002) The role of concomitant headache types and non-headache co-morbidities in the underdiagnosis of migraine. Neurology 58(9 Suppl 6):S3-S9

2. Cady RK, Schreiber CP (2002) Sinus headache or migraine? Considerations in making a differential diagnosis. Neurology 58(Suppl 6):S10-S14
3. Lanza DC, Kennedy DW (1997) Adult rhinosinusitis defined. Otolaryngol Head Neck Surg 117:1-7

4. American Academy of Pediatrics Subcommittee on Management of Sinusitis and Committee on Quality Improvement (2001) Clinical practice guideline: management of sinusitis. Pediatrics 108:798-808

5. Headache Classification Committee of the International Headache Society (1998) Classification and diagnostic criteria for headache disorders, cranial neuralgias and facial pain. Cephalalgia 8(Suppl 7):1-96

6. Headache Classification Committee of the International Headache Society (2004) The international classification of headache disorders: 2nd edition. Cephalalgia 24(Suppl 1):9-160

7. Winner P, Martinez W, Mante L et al (1995) Classification of pediatric migraine: proposed revisions to the IHS criteria. Headache 35:407-410

8. Maytal J, Young M, Schechter A et al (1997) Pediatric migraine and the International Headache Society (IHS) criteria. Neurology 48:602-607

9. Seshia S, Wolstein J, Adams C et al (1994) International Headache Society criteria and childhood headache. Dev Med Child Neurol 36:419-428

10. Gallai V, Sarchielli P, Carboni F et al (1995) Applicability of the 1988 IHS criteria to headache patients under the age of 18 years attending 21 Italian headache clinics. Headache 35:146-153

11. deGrauw TJ, Hershey AD, Powers SW et al (1999) Diagnosis of migraine in children attending a pediatric headache clinic. Headache 39:481-485

12. Rossi LN, Cortinovis I, Menegazzo L et al (2001) Classification criteria and distinction between migraine and tension-type headache in children. Dev Med Child Neurol 43:45-51

13. Wöber-Bingöl Ç, Wöber C, Wagner-Ennsgraber C et al (1996) IHS criteria for migraine and tension-type headache in children and adolescents. Headache 36:231-238

14. Cady RK, Schreiber CP, Billings C (2001) Subjects with self decribed "sinus" headache meet IHS diagnostic criteria for migraine. Cephalalgia 21:298

15. Schreiber CP, Cady RK, Billings C (2001) Oral sumatriptan for self described "sinus" headache. Cephalalgia 21:298

16. Meggs WJ (1995) Neurogenic switching: a hypothesis for mechanism for shifting the site of inflammation in allergy and chemical sensitivity. Environ Health Perspect 103:54-56

17. Blumenthal HJ (2001) Headaches and sinus disease. Headache 41:883-888

18. Tarabichi M (2000) Characteristics of sinus-related pain. Otolaryngol Head Neck Surg 122:842-847

19. Kenny TJ, Duncavage J, Bracikowski J et al (2001) Prospective analysis of sinus symptoms and correlation with paranasal computed tomography scan. Otolaryngol Head Neck Surg 125:40-43 